\title{
Surgical Versus Non-Surgical Treatment for Traumatic Esophageal Perforation in Children: A Systematic Review
}

\author{
Abdulwahhab Al Jubab ${ }^{\mathrm{a}, \mathrm{c}}$, Ilhama A. Jafarli ${ }^{\mathrm{a}}$, Tariq Al Tokhais ${ }^{\mathrm{b}}$
}

\begin{abstract}
The study design of this paper is a systematic review of literature published in the recent 10 years. Esophageal perforations in children have long been a topic of debate. The management protocols are chiefly governed by symptom severity, perforation site, time elapsed since perforation and cause of perforation. Esophageal perforations in pediatric group of patients can be iatrogenic or traumatic. The aim to conduct the study was to assess the benefits and timely management of surgical versus non-surgical treatment for pediatric patients with traumatic esophageal perforation. The two research questions below were determined. We systematically reviewed retrospective serial studies assessing the medical treatment compared to the surgical interventions for the traumatic esophageal perforation in children. We searched MEDLINE, EMBASE, Cochrane Central Register of Controlled Trials, and Cochrane Database of Abstracts of Reviews of Effects, as well as foreign literature with English translations. No randomized controlled trial studies had been conducted in children with esophageal perforation. Information on patients' age, comorbidities, methods of treatment, and effects on mortality, morbidity were extracted. Three independent reviewers selected the articles for analysis after screening the titles, abstracts, and full texts, then extracted data and graded the quality of each paper according to the Grading of Recommendations, Assessment, Development, and Evaluation (GRADE) criteria. Specific clinical questions were as follows. 1) In patients with early diagnosis of esophageal perforation, what are the indications for treatment (surgery versus conservative management) and its impact on prognosis? 2) In patients with delayed diagnosis, what are the indications for treatment (surgery versus conservative management) and its impact on mortality and morbidity rate? A total of 66 abstracts were identified using various keywords. Nine retrospective articles (level III) were eligible for inclusion, involving a total of 77 cases of esophageal perforation in pediatric patients. Non-operative management is recommended for
\end{abstract}

Manuscript accepted for publication March 15, 2016

aPediatric Surgery Department, King Fahad Medical City, Children's Hospital, PO Box 59046, Riyadh 11525, Saudi Arabia

bDepartment of Surgery, King Saud University, College of Medicine, PO Box 2454, Riyadh 11452, Saudi Arabia

${ }^{c}$ Corresponding Author: Abdulwahhab Al Jubab, Pediatric Surgery Department, King Fahad Medical City, Children's Hospital, PO Box 59046, Riyadh 11525, Saudi Arabia. Email: aaljubab@kfmc.med.sa

doi: http://dx.doi.org/10.14740/jcs296w perforations diagnosed within 24 - 48 hours in a stable patient with contained leakage, but hemodynamically unstable patients with a contained perforation, ongoing leakage and early diagnosed have a higher chance of successful primary repair, whereas delayed ones require conservative treatment.

Keywords: Esophagus; Contained perforation; Ongoing leakage; Surgery; Conservative management; Endoscopy; Pediatric patients

\section{Introduction}

Esophageal perforation is a rare and potentially life-threatening condition. Early clinical suspicion and imaging is important for case management to achieve a good outcome [1]. Esophageal perforation is most commonly iatrogenic in origin with nasogastric tube insertion, stricture dilation, retrieval of the foreign bodies using esophagoscopy and endotracheal intubation, being the most frequent sources of the injury in infants and children. Clinical presentation depends on whether the cervical, thoracic, or abdominal esophagus is injured. Any patient complaining of chest pain after an upper endoscopy has esophageal perforation until proven otherwise [2]. Catheter interventions in the upper aerodigestive tract, such as the placement of orogastric catheter, nasogastric catheter and the procedure of oro-naso-pharyngeal suction are common medical practices in critically ill patients in the intensive care units that may concurrently have more iatrogenic problems [3]. Ingestion of foreign bodies, mainly button batteries, also contributes to the severe esophageal injury in a group of patients ranged between 6 months and 3 years of age [4]. Signs and symptoms included tachycardia, dysphagia, dyspnea, fever, cyanosis, abdominal pain, chest pain, and subcutaneous emphysema [5].

Several factors, including the difficulty of accessing the esophagus, the lack of a serosal layer, the unusual blood supply of the organ and the proximity of vital structures, all contribute to this condition's high morbidity and to a mortality rate [68]. In addition, the diversity of clinical symptoms and signs combined with a lack of individual experience regarding this particular condition may impede rapid identification of this potentially hazardous situation. Accordingly, delayed diagnostic workup may hinder timely and appropriate treatment with a negative effect on patient outcome [9].

Children with esophageal perforation should receive fluid resuscitation and administration of broad-spectrum antibiot- 


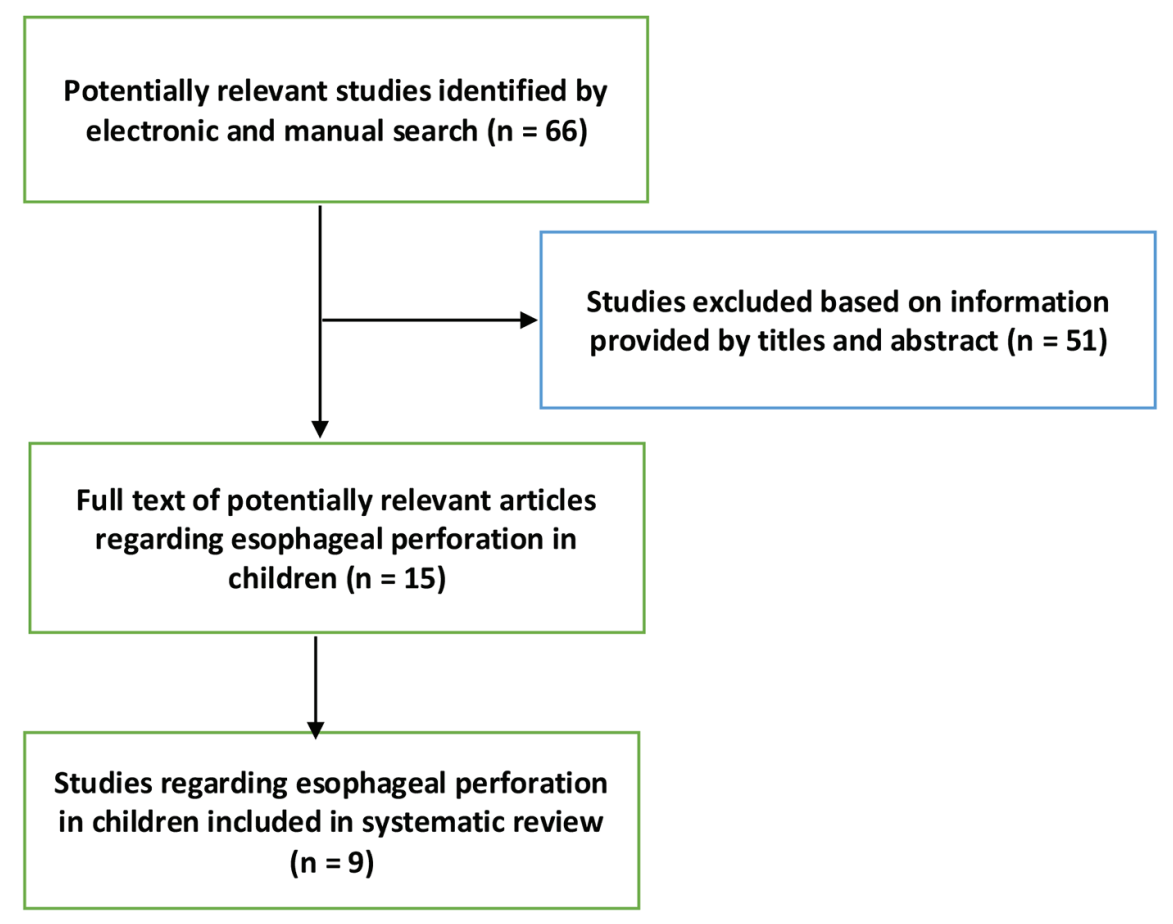

Figure 1. Flow diagram showing identification of studies included in the review.

ics to cover gram-positive, gram-negative, and anaerobic organisms. Esophageal injuries with ongoing leakage should be repaired as soon as possible, since delay in repair results in increased mortality. Perforations with contained leak in a stable patient on esophagography may be successfully managed non-operatively [10].

The rarity of this emergency makes it difficult for a physician to obtain extensive individual clinical experience; it is also challenging to obtain firm scientific evidence that informs patient management and clinical decision making. Improved attention to non-specific symptoms and signs and early diagnosis based on imaging may translate into better outcomes for pediatric patients.

Despite numerous retrospective serial reports on conservative versus surgical treatment of esophageal perforation in children, there exist no randomized controlled comparisons of clinical efficacy and safety between procedures. In addition, due to the heterogeneity of study designs, inconsistent reporting of complications, and the use of different grading scales for early and delayed diagnosis, it was not possible to perform a meta-analysis using the retrospective studies. Therefore, we endeavored to perform a quantitative systematic review of the current literature published in the recent 10 years to evaluate the clinical efficacy between surgical and non-surgical treatment in pediatric patients with esophageal perforation.

\section{Methods}

Two clinically relevant questions below were determined and a systematic review of related literature published in the re- cent 10 years was conducted using MEDLINE, EMBASE, Cochrane Central Register of Controlled Trials, and Cochrane Database of Abstracts of Reviews of Effects, as well as foreign literature with English translations. Specific clinical questions were as follows: 1) In patients with early diagnosis of esophageal perforation, what are the indications for treatment (surgery versus conservative management) and its impact on prognosis? 2) In patients with delayed diagnosis, what are the indications for treatment (surgery versus conservative management) and its impact on mortality and morbidity rate?

\section{Search criteria}

We used the search terms that included "esophag*", "perforation*", "child*" and "surg*" to search literature from MEDLINE. The following terms would be contained: "esophagus", "esophageal", "perforation", "child", "children", "surgery", and "surgical".

Criteria for possible inclusion are as follows: 1) articles published in the recent 10 years, 2) all articles in English or with an English translation, 3$)$ pediatric age group ( 0 - 14 years old), 4) articles describing surgical treatment of iatrogenic esophageal perforation in children, 5) articles evaluating the conservative management in pediatric patients with esophageal perforation, and 6) articles with non-homogeneous pathology (e.g., trauma and another comorbidity in the same series). Exclusion criteria include the following: 1) spontaneous perforations of the esophagus, and 2) age group above 14 years old.

Studies were reviewed using a standardized data collection form. The type of study (retrospective) was noted. Data 
Table 1. Characteristics of Included Studies

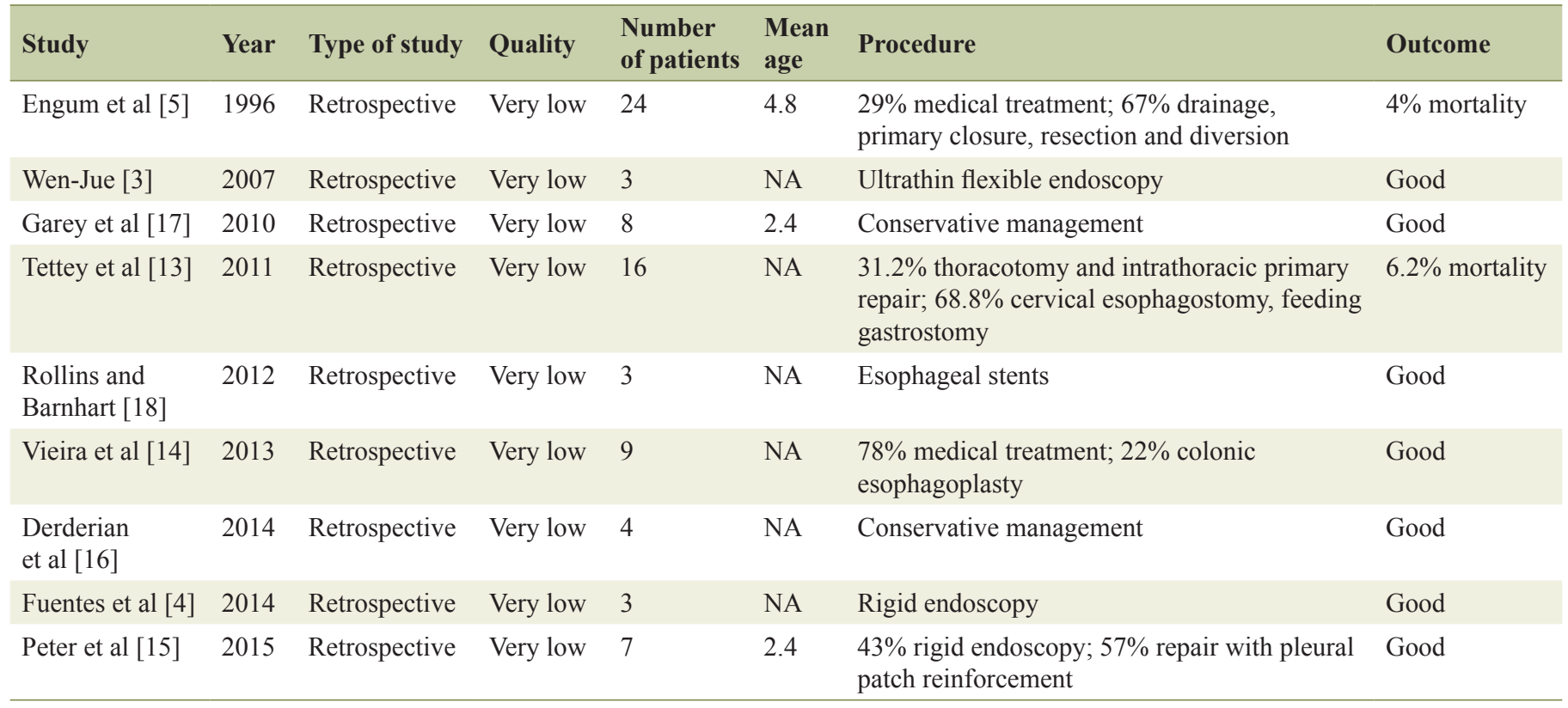

including surgery technique, the total number of patients, and mean age were collected. To avoid duplicate records of patients' data, each group or institution was limited to one study in the systematic review.

The quality of evidence for each article was evaluated as high, moderate, low, or very low. The group then went through decision-making process using a modified Delphi technique to arrive at treatment recommendations related to the key clinical questions. This process and the strength of the recommendation were based on the Grading of Recommendations, Assessment, Development, and Evaluation (GRADE) method [11, 12]. These articles were evaluated independently by the authors according to the GRADE criteria.

\section{Results}

A total of 66 abstracts were identified using various keywords. All abstracts were screened, and 51 articles were excluded as obviously unrelated. The full texts of 15 papers were screened and nine papers were identified to meet the inclusion criteria, retrospective articles (level III). The details of article selection were presented in Figure 1 and Table 1 [3, 4, 13-18].

\section{Discussion}

Non-operative treatment is appropriate for many pediatric patients with iatrogenic perforation, i.e. perforation after dilatation, button battery ingestion, coin ingestion, nasogastric tube placement, insertion of a transesophageal echocardiogram probe and leak after stricture resection (Fig. 2).

In infants and children, plain chest films and esophagography, using water soluble contrast may assist in making the diagnosis. Free perforation and hemodynamic lability mandate a more aggressive surgical approach for wide drainage of the mediastinum and pleural spaces [2].

In patients with injury to the esophageal wall and contained leakage without systemic symptoms of infection and compromised circulation, careful observation, nil per mouth, appropriate treatment with intravenous broad-spectrum antibiotics and proton pump inhibitors (PPIs) and nutritional support may be sufficient for successful treatment. Children with esophageal perforation should receive fluid resuscitation and administration of broad-spectrum antibiotics to cover grampositive, gram-negative, and anaerobic organisms (e.g., piperacillin/tazobactam for patients with penicillin allergy, clindamycin and gentamicin) [10].

Primary repair of esophageal perforation is possible, especially in patients admitted to the hospital within $24 \mathrm{~h}$ of the event. Morbidity and mortality are directly related to delays in diagnosis and therapy. Most cases of esophageal perforation in children can be closed primarily and the esophagus salvaged despite delayed presentation. The mortality rate in children with esophageal perforation (4\%) is significantly less than that for adults (25-50\%) [5].

Incidence of button battery ingestion is increasing in the last years and the early diagnosis, when they become lodged in esophagus, is of capital importance to diminish the risk of potential fatal complications. Endoscopic removal and a close follow-up by a multi-disciplinary group of physicians are essential to deal with both early and late complications [5]. Esophageal perforation is a rare complication of transesophageal echocardiogram probe placement as well. Complications include pneumothorax, pneumomediastinum with associated infection, pseudodiverticulum formation and esophageal obstruction. Non-operative management with broad-spectrum antibiotics and nothing per mouth is a safe and effective treat- 


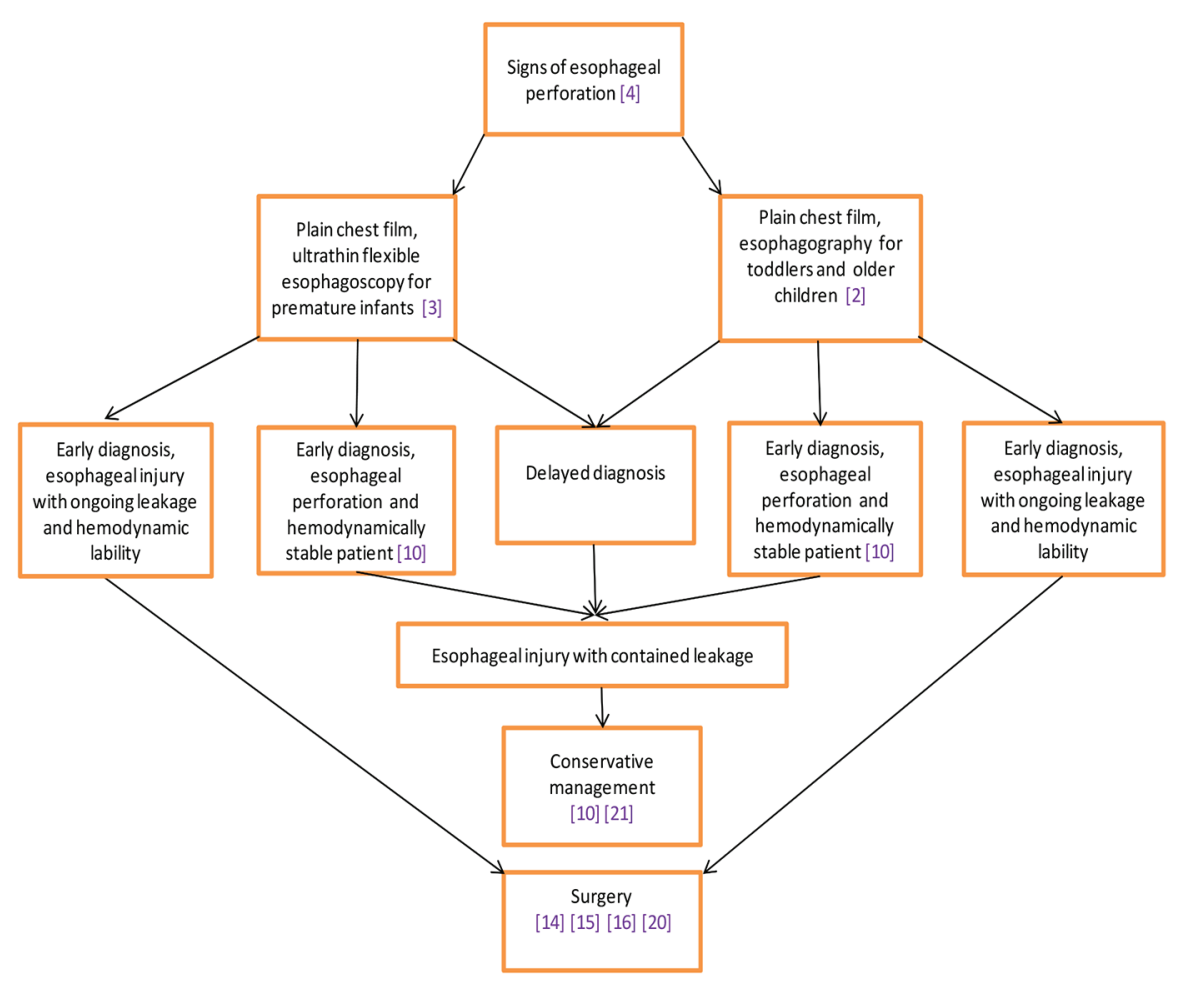

Figure 2. Algorithm of the management of the traumatic esophageal perforation in children.

ment strategy for infants with non-complicated perforations resulting from a transesophageal echocardiogram probe [16].

Recent reports emphasize a shift in treatment strategies over the last few decades, with non-operative approaches, such as percutaneous drainage of pleural effusions, collections or abscesses, becoming more common [5]. In addition, there is a growing use of temporary endoscopic esophageal stents to seal esophageal leakage and to recover gastrointestinal continuity [18]. The treatment, being individual to each patient, is mostly based on the time of presentation. Early diagnosed cases have a higher chance of successful primary repair, whereas delayed ones require conservative treatment. The majority of late-presented or late-diagnosed cases can be successfully managed conservatively [19].

\section{Conclusion}

In conclusion, non-operative management is recommended for perforations diagnosed within $24-48 \mathrm{~h}$ in a stable patient with no mediastinitis or empyema. The delay of the diagnosis is associated with a mortality which can oscillate between $4 \%$ and $20 \%$. However, it is necessary to perform a controlled study to compare the clinical efficiency between the two methods of treatment.

\section{Competing Interests}

The authors declare that they have no competing interests.

\section{References}

1. Soreide JA, Viste A. Esophageal perforation: diagnostic work-up and clinical decision-making in the first 24 hours. Scand J Trauma Resusc Emerg Med. 2011;19(66.

2. Gander JW, Berdon WE, Cowles RA. Iatrogenic esophageal perforation in children. Pediatr Surg Int. 2009;25(5):395-401.

3. Soong WJ. Endoscopic diagnosis and management of iatrogenic cervical esophageal perforation in extremely premature infants. J Chin Med Assoc. 2007;70(4):171-175.

4. Fuentes S, Cano I, Benavent MI, Gomez A. Severe esophageal injuries caused by accidental button battery ingestion in children. J Emerg Trauma Shock. 2014;7(4):316321.

5. Engum SA, Grosfeld JL, West KW, Rescorla FJ, Scherer LR, Vaughan WG. Improved survival in children with esophageal perforation. Arch Surg. 1996;131(6):604-610; discussion 611.

6. Ryom P, Ravn JB, Penninga L, Schmidt S, Iversen MG, Skov-Olsen P, Kehlet H. Aetiology, treatment and mortality after oesophageal perforation in Denmark. Dan Med Bull. 2011;58(5):A4267.

7. Bhatia P, Fortin D, Inculet RI, Malthaner RA. Current concepts in the management of esophageal perforations: a twenty-seven year Canadian experience. Ann Thorac Surg. 2011;92(1):209-215.

8. Vidarsdottir H, Blondal S, Alfredsson H, Geirsson A, Gudbjartsson T. Oesophageal perforations in Iceland: a whole population study on incidence, aetiology and sur- 
gical outcome. Thorac Cardiovasc Surg. 2010;58(8):476480.

9. Onat S, Ulku R, Cigdem KM, Avci A, Ozcelik C. Factors affecting the outcome of surgically treated noniatrogenic traumatic cervical esophageal perforation: 28 years experience at a single center. J Cardiothorac Surg. 2010;5(46.

10. Eroglu A, Can Kurkcuogu I, Karaoganogu N, Tekinbas C, Yimaz O, Basog M. Esophageal perforation: the importance of early diagnosis and primary repair. Dis Esophagus. 2004;17(1):91-94.

11. Brozek JL, Akl EA, Alonso-Coello P, Lang D, Jaeschke R, Williams JW, Phillips B, et al. Grading quality of evidence and strength of recommendations in clinical practice guidelines. Part 1 of 3 . An overview of the GRADE approach and grading quality of evidence about interventions. Allergy. 2009;64(5):669-677.

12. Schunemann HJ, Jaeschke R, Cook DJ, Bria WF, El-Solh AA, Ernst A, Fahy BF, et al. An official ATS statement: grading the quality of evidence and strength of recommendations in ATS guidelines and recommendations. Am J Respir Crit Care Med. 2006;174(5):605-614.

13. Tettey M, Edwin F, Aniteye E, Sereboe L, Tamatey M, Entsua-Mensah K, Kotei D, et al. Management of intrathoracic oesophageal perforation: analysis of 16 cases.
Trop Doct. 2011;41(4):201-203.

14. Vieira E, Cabral MJ, Goncalves M. [Esophageal perforation in children: a review of one pediatric surgery institution's experience (16 years)]. Acta Med Port. 2013;26(2):102-106.

15. Peters NJ, Mahajan JK, Bawa M, Chabbra A, Garg R, Rao KL. Esophageal perforations due to foreign body impaction in children. J Pediatr Surg. 2015;50(8):12601263.

16. S. Christopher Derderian, Mark L. Wulkan, Kirk R. Kanter, Steven Tosone, Matthew S. lifton. Management of esophageal perforation in infants resulting from transesophageal echocardiogram probes. J Ped Surg Case Reports. 2014;2(6):328-330.

17. Garey CL, Laituri CA, Kaye AJ, Ostlie DJ, Snyder CL, Holcomb GW, 3rd, St Peter SD. Esophageal perforation in children: a review of one institution's experience. J Surg Res. 2010;164(1):13-17.

18. Rollins MD, Barnhart DC. Treatment of persistent esophageal leaks in children with removable, covered stents. J Pediatr Surg. 2012;47(10):1843-1847.

19. Emil Mammadov, Altan Alim, Mehmet Elic, evik, Sinan Celayir. Traumatic upper cervical esophageal perforation in childhood with door handle. Annals of Pediatric Surgery. $2011 ; 7: 23-24$. 